\title{
Analysis on energy saving and environmental protection in building design
}

\author{
Zhaozhiyuan $^{1, \mathrm{a}}$ \\ ${ }^{1}$ School of Civil Engineering and Architecture, Wuhan University of Technology, Wuhan,HuBei Province,430070, China
}

\begin{abstract}
The goal of the concept of environmental protection and energy conservation is to promote the development of resource economy. In the process of my country's economic development, the ecological environment has been destroyed and the resource situation is not optimistic. Implementing the concept of environmental protection and energy conservation in the design of building water supply and drainage can increase resource utilization, weaken the damage to the environment by project construction, reduce project costs, and alleviate the current situation of resource shortage in my country. Combining this situation, this article focuses on analyzing the importance of the application of energy-saving and water-saving technologies in the construction of building water supply and drainage and the specific application of energy-saving and water-saving technologies.
\end{abstract}

\section{Introduction}

In recent years, with the continuous development of the construction industry, medium and large buildings in cities have gradually increased. As a high water consumption industry, the construction industry must proceed from the reality of building water supply and drainage in the construction process to enhance the application of energy-saving and environmental protection technologies in building design. When designing building water supply and drainage, it is necessary to scientifically and rationally use the design concept of energy saving and environmental protection, and continuously optimize and adjust the original design plan. Applying the concept of environmental protection and energy saving to the design of building water supply and drainage can effectively promote the sustainable development of the construction industry and realize the healthy development of construction enterprises ${ }^{[1]}$.

\section{Common problems in building water supply and drainage design}

Due to the design concept, project cost, construction level and other reasons of the building water supply and drainage system, there may be many problems in the process of use, such as unreasonable pipeline layout and difficulty in underground sewage discharge.

(1) Water consumption problems caused by pipes, valves and other accessories

In the process of building design and construction, when the construction process is unreasonable or the design does not conform to the actual situation, the problem of water waste will be triggered. In the design of building water supply and drainage, inadequate connection of valves and fittings, or leakage of pipes, will cause waste of water resources.

(2) Water waste caused by pipeline overpressure

When the required flow rate in the actual situation is greater than the rated flow rate set by the sanitary appliances in the construction project, the pressure of the water supply fittings will be greater than the outflow head. This is easy to produce overpressure outflow, which seriously affects the water use of high-rise residents and the performance of construction projects.

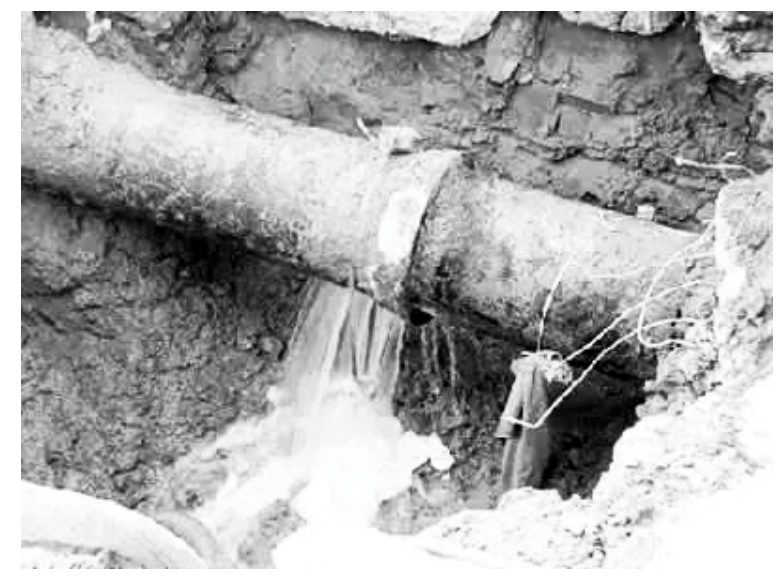

Figure 1. Burst of water supply pipe

(3) Low utilization rate of renewable water resources During the actual use of building water supply and drainage, many buildings' rainwater and domestic water are directly drained from the drainage pipes, and they are not used twice or more. This causes a waste of water resources and reduces the utilization rate of renewable

a Zhaozhiyuan: zzy1208299@163.com 
water resources in the building's water supply and drainage system.

(4) Energy-saving buildings are not widely used

On the one hand, my country has a relatively short period of time for energy-saving applications of building water supply and drainage. This makes the awareness of energy conservation and environmental protection in the building design process relatively poor, and it is difficult to implement the design concept of energy conservation and environmental protection. On the other hand, the development of energy-saving building materials in my country is insufficient. Therefore, the energy-saving design of building water supply and drainage is mostly formal, which makes the practicality and energy-saving of the building conflict, and the energy-saving effect is not obvious.

(5) The actual construction of the construction project lacks perfect construction specifications, and the construction technology is also uneven.

There are many factors that bring quality problems to construction projects. For example, there is a lack of installation personnel with a high level of professionalism. Most workers have not received professional training or received correct guidance from experienced technicians. Therefore, it is difficult to carry out construction in strict accordance with the standards, leading to problems in construction quality. In addition, the lack of a complete construction supervision system is also a key issue leading to quality degradation. The difference from other construction methods is that most construction units mistakenly believe that the main body of the construction project is the civil part, and the construction of the water supply and drainage project is carried out according to the supporting standards, and the management personnel also occasionally participate in the construction process. Therefore, the construction quality is generally defective, and it is difficult to complete the rectification work in a timely and effective manner, which has a serious impact on the quality of the water supply and drainage project and hinders the smooth development of the acceptance work.

(6) The construction quality and supervision lack management strength

Continuously improving the level of construction technology will help to effectively control the construction details and maintain a high construction quality. However, most of the constructions are not equipped with professional construction personnel in the water supply and drainage construction, but simply outsource the water supply and drainage project. For ordinary construction teams, professional technicians are not arranged for guidance during the actual construction process, and some construction procedures and quality acceptance reports are not standardized. At the same time, some construction workers lack a correct working attitude, and blindly carry out construction work according to their own ideas during construction, and fail to strictly follow the specific requirements of the drawing design, which affects the construction quality. At the same time, engineering supervision departments lack sufficient control efforts. Most of the supervision departments regard the control of the overall quality of buildings as the key to quality control.
They lack a high degree of attention to the installation of water and electricity, and even think that these are only auxiliary content in the construction of the project. The quality of the water supply and drainage system that has completed the construction work has not been tested, leaving many hidden quality problems in the pipeline.

\section{The necessity of energy-saving design for building water supply and drainage}

The significance of energy saving and environmental protection in house building design is to be able to use key cards. Through the energy-saving and environmentallyfriendly design of building water supply and drainage, and good energy-saving and environmental protection measures, pollution prevention and control can be effectively ensured, and sustainable development can be achieved. If the architectural design can achieve energy saving, environmental protection and reduce consumption, this will greatly promote the protection of the environment $[2-4]$.

(1) Environmental protection needs

Water resources play an irreplaceable role in human survival and development. The application of energysaving and water-saving technologies for building water supply and drainage can effectively reduce the waste of water resources, give full play to the maximum utilization value of water resources, and promote the sound development of the entire construction industry and the entire society.

(2) Promote economic development

my country is a big energy consuming country. Under the current development background of energy shortage, it is particularly important to integrate the concept of energy saving and emission reduction into the design of building water supply and drainage. This not only has a positive effect on the conservation of energy resources, but also has a positive role in promoting economic development. It will also play a positive role in my country's economic development and promote my country's economic prosperity.

\section{Methods of building energy-saving technology}

To achieve energy conservation and environmental protection in the design of building water supply and drainage, we need to consider from different angles. This section lists several common building energy-saving technologies for reference.

(1) Collection and utilization of rainwater

Analyzing the spatial distribution of precipitation throughout the country, the rainfall is gradually decreasing from the southeast coastal areas to the northwest inland areas. Especially in the southeast coastal area, the annual precipitation can reach $2000-3150 \mathrm{~mm}$. In the building water supply and drainage system, the collected rainwater is used for many purposes. In addition to the supply of drinking water, rainwater collection and utilization devices can effectively use it in the supply of other 
domestic water. In architectural design, not only must collect rainwater from roof, roof and ground, but also deal with it in time after collection. According to the water quality of runoff, rainwater resources are classified and used. The drainage pipes of the top users should use side wall rainwater buckets to increase the collection area of rainwater. For the discharge of rainwater from the balcony, a non-water-sealed floor drain is used to collect it.

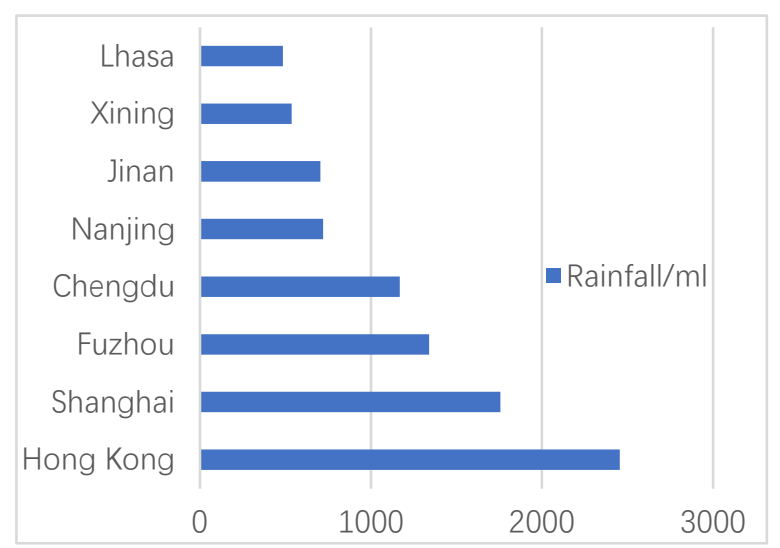

Figure 2. Rainfall map of some capital cities

(2) Utilization of middle water resources

In the design of building water supply and drainage, reclaimed water plays an extremely important role. The survey shows that $62 \%$ of building drainage is domestic wastewater discharged from residential buildings. The collected and treated wastewater can be converted into reclaimed water for vehicle cleaning, sanitary appliance cleaning and irrigation, etc., reducing water resources. waste. Reclaimed water treatment technology includes physicochemical filtration method, microbial adsorption method and membrane filtration method.

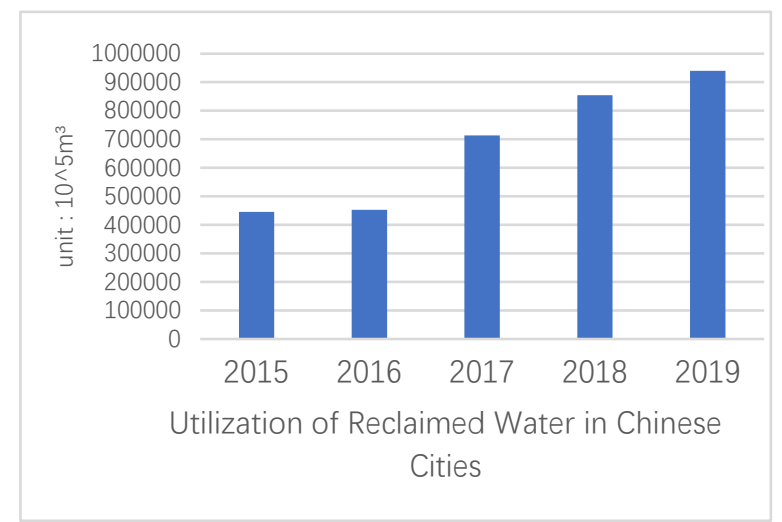

Figure 3. Changes in China's urban reclaimed water utilization from 2015 to 2019

(3) Optimized design of water supply and drainage pipelines

In the design of water supply pipes, it is necessary to consider that the plastic pipes used have the advantages of corrosion resistance and easy cleaning, but they will expand due to heat ${ }^{[5,6]}$. When the pipeline expands due to heat or the quality of the pipe is not up to the standard, it is prone to overflow problems, which increases the chance of wasting water resources. Therefore, in the design of water supply pipelines, rational and optimized design lines should be selected to reduce the consumption of pipes.
In the design of drainage piping, attention should be paid to strengthening effective quality control in the link of material selection. In the process of pipeline design, a reasonable installation method of pipe installation can optimize the design of the drainage pipeline. For example, the method of combining pipe grooves and pipe wells, among which the spiral and core foaming methods can not be installed, can achieve the goal of energy saving and emission reduction, and minimize noise.

The metal pipeline is hard and stable, with remarkable mechanical strength, compressive performance, seismic performance and impermeability. The inner wall of the pipeline is flat and smooth, and the water flow resistance is small. The metal pipeline has long sections, few joints, simple interfaces and low construction difficulty. However, metal pipes also have defects and deficiencies, such as strong oxidation reaction and polluting water sources; weak chemical resistance to corrosion, and the metal surface layer is easy to fall off, which has a greater impact on the use of pipes.

The plastic pipe is melt-processed and synthesized by resin and additives. It has stable chemical properties, will not be affected by the medium and soil, has strong corrosion resistance, and has low thermal conductivity. The inner wall of the pipeline is flat and smooth, has good hydraulic performance, and has a small amount of fouling, which is convenient for installation and transportation. In addition, the plastic pipe has good cold bending performance and can be coiled, thereby reducing the number of pipe joints and enhancing the sealing of the pipe. However, plastic pipes also have shortcomings, insufficient compression resistance, flame retardancy, and easy deformation under pressure. After the pipe is burned, it releases smoke and toxic gases, has a high thermal expansion coefficient, insufficient sound insulation and heat insulation, and is easily aging and damaged.

The composite pipe is composed of a protective layer and a support layer, which not only has the strength and rigidity advantages of metal pipes, but also has the corrosion resistance advantages of non-metal pipes. However, composite pipes also have shortcomings and shortcomings. Due to the large difference in thermal expansion coefficients of different materials, when the medium temperature and ambient temperature change significantly, it will not only cause the pipeline to disconnect and reduce the quality of the pipeline, but also cause the pipe connection part to be unstable, and the potential risk of falling off is large .

\section{Design trend of building water supply and drainage}

With the development of technology, materials and other fields, the water supply and drainage system of high-rise buildings is gradually developing in the direction of smarter, more energy-saving, more economical, more beautiful, and more concise.

(1) Intelligent. The water supply and drainage system of high-rise buildings is very complicated. Different areas, different pipelines, and different functions of water flow characteristics, flow rates, and water pressure 
requirements are different. It is costly and difficult to control these factors by traditional methods to achieve the most ideal state. With the development of intelligent technology, it has become possible to use intelligent control technology to automatically adjust the water supply and drainage system. In the near future, the intelligentization of water supply and drainage systems will be the main development direction.

(2) Pay more attention to energy saving. Considering environmental, resource, economic and other factors, the development and application of more energy-efficient water supply and drainage equipment and technologies is the general trend.

(3) More economical. With the gradual shortage of water resources, and the high cost of water supply in highrise buildings, the water supply per unit time is limited, and effective water conservation has become a problem that must be faced. Such as adopting water-saving faucets, optimizing the design of reclaimed water system, and adopting variable frequency water pumps in a wide range. Combining intelligent control, the development of more accurate water-saving equipment will be one of the research focuses.

(4) More concise. The space resources of high-rise buildings are limited, and the water supply and drainage system is very complicated, which increases operation and maintenance costs. By adopting new technologies and methods, making the water supply and drainage system simple, efficient and less noise has been the main development direction and key concern.

\section{Conclusion}

(1) Many problems are contained in the energy-saving design of building water supply and drainage

(2) The significance of energy saving and environmental protection in building design is to be able to use key cards. The energy-saving and environmentallyfriendly design of building water supply and drainage will reduce consumption and greatly promote the protection of the environment.

(3) In the design of building water supply and drainage, methods such as rainwater collection, utilization of water resources, optimization of water supply and drainage design, and optimization of pipes and pipes can be used.

\section{References}

1. high performance buildings: the link between occupant knowledge of passive design systems, corresponding behaviors occupant comfort and environmental satisfaction $[\mathrm{J}]$. Building and Environment, 84: 114-124 (2015) .

2. SAHAR Z, HEBA E . Towards energy-efficient retrofitof council housing in London: assessing the impact of occupancy and energy-use patterns on building performance $[\mathrm{J}]$. Energy and Buildings, 174: 672-681 (2018).
3. MOON J W, CHUNG S K . Development of a thermal control algorithm using artificial neural network models for improved thermal comfort and energy efficiency in accommodation buildings[J] . Applied Thermal Engineering, 103: 1135-1144 (2016) .

4. Zhu Sicheng. The underground comprehensive pipe gallery in Tokyo City [J] . China Water \& Wastewater, 21 ( 3) : 102- 103 (2015).

5. Li Chunmei . Life cycle management: the Singapore model of underground integrated pipe gallery

[J ] . China Exploration \& Design : 72-75 (2016)

6. Zheng Fengshou, Tao Weixiang, Pan Liangbo, et al . $\mathrm{R}$ esearch on the intelligent management platform of urban underground pipelines $[\mathrm{J}]$. Chinese Journal of Underground Space and Engineering 11 ( Supp . 2) : 378-3 (2016) 